\title{
Serrated colorectal polyps in inflammatory bowel disease
}

\author{
Huaibin M Ko ${ }^{1,4}$, Noam Harpaz ${ }^{1,2,4}$, Russell B McBride ${ }^{1,3}$, Miao Cui ${ }^{1}$, Fei Ye ${ }^{1}$, David Zhang ${ }^{1}$, \\ Thomas A Ullman ${ }^{2}$ and Alexandros D Polydorides ${ }^{1,2}$ \\ ${ }^{1}$ Department of Pathology, Icahn School of Medicine at Mount Sinai, New York, NY, USA; ${ }^{2}$ Department of \\ Medicine (Gastroenterology), Icahn School of Medicine at Mount Sinai, New York, NY, USA and ${ }^{3}$ Institute for \\ Translational Epidemiology, Icahn School of Medicine at Mount Sinai, New York, NY, USA
}

\begin{abstract}
Serrated colorectal polyps, which, besides hyperplastic polyps, comprise sessile serrated adenomas/polyps and traditional serrated adenomas, are presumptive precursors of at least $20 \%$ of sporadic colorectal carcinomas; however, their significance in patients with inflammatory bowel disease is unclear. We retrospectively evaluated 78 serrated polyps, removed over a 14-year period from 6602 inflammatory bowel disease patients undergoing endoscopic surveillance, with respect to morphologic, clinicopathologic, and molecular features, and compared rates of advanced neoplasia (high-grade dysplasia and carcinoma) development following the index serrated polyp diagnosis to reference inflammatory bowel disease cohorts without serrated polyps. Serrated polyps negative for dysplasia, which morphologically resembled sporadic sessile serrated adenoma/polyps, occurred mainly in females, in the proximal colon, and contained BRAF mutations. Serrated polyps with low-grade dysplasia resembled sporadic traditional serrated adenomas and occurred mainly in males, in the distal colon, and contained KRAS mutations. Serrated polyps indefinite for dysplasia were morphologically heterogeneous, but similar to serrated polyps positive for low-grade dysplasia with respect to male predominance, left-sided location, and KRAS mutation rates. Rates of prevalent neoplasia associated with serrated polyps positive for lowgrade dysplasia, indefinite for dysplasia, and negative for dysplasia were 76,39 , and $11 \%$, respectively $(P<0.001)$. Actuarial 10-year rates of incident advanced neoplasia after an initial diagnosis of serrated polyp positive for low-grade dysplasia, indefinite for dysplasia, and negative for dysplasia were 17,8 , and $0 \%$, respectively, the first and last being significantly different $(P=0.02)$ and comparable to those of corresponding reference populations of inflammatory bowel disease patients with and without low-grade dysplasia at baseline, respectively. We conclude that in serrated polyps from inflammatory bowel disease patients, dysplasia grade correlates with morphology, sex, anatomic location, BRAF and KRAS mutation status, prevalent conventional neoplasia, and rates of advanced neoplasia development.
\end{abstract}

Modern Pathology (2015) 28, 1584-1593; doi:10.1038/modpathol.2015.111; published online 25 September 2015

Recognition of the serrated neoplasia pathway to colorectal carcinoma has enhanced our understanding of sporadic carcinogenesis in the general population. Serrated polyps, which encompass, besides hyperplastic polyps, sessile serrated adenomas/ polyps and traditional serrated adenomas, have been

Correspondence: Dr AD Polydorides, MD, PhD, Department of Pathology, Icahn School of Medicine at Mount Sinai, One Gustave L. Levy Place, Box 1194, New York, NY 10029, USA.

E-mail: alexandros.polydorides@mountsinai.org

${ }^{4}$ These authors contributed equally to this work.

Data in this study have been partially presented at the 2013 and 2015 annual meetings of the United States and Canadian Academy of Pathology.

Received 5 June 2015; revised 14 August 2015; accepted 15 August 2015; published online 25 September 2015 implicated in the pathogenesis of at least $20 \%$ of sporadic colorectal carcinomas. ${ }^{1,2}$ Sessile serrated adenoma/polyps are usually located in the proximal colon, predominate in women, and harbor BRAF mutations, whereas traditional serrated adenomas are usually left sided, occur more often in men, and contain KRAS mutations. ${ }^{3-5}$ The significance of serrated polyps in the general population with respect to carcinoma risk stratification, endoscopic surveillance, and management is becoming increasingly clear and has recently been incorporated into clinical consensus guidelines. ${ }^{6-8}$

Patients with extensive, longstanding colonic inflammatory bowel disease, mainly ulcerative and Crohn's colitis, face an increased risk of developing colorectal cancer compared with the general population. ${ }^{9,10}$ Surveillance colonoscopy is advo- 
cated and widely practiced in these patients as a means of reducing cancer mortality by permitting early detection and management of neoplasia in the form of dysplasia or early, curable carcinoma. ${ }^{9,11}$ The histologic grading, natural history, clinical implications, and molecular basis of dysplasia in inflammatory bowel disease have been studied extensively and provide essential guidance in this endeavor. ${ }^{9,11-13}$

Nonetheless, recognition of the full range of histologic lesions with malignant potential in the setting of inflammatory bowel disease is incomplete. Although several prior studies have described serrated colorectal lesions in patients with inflammatory bowel disease, ${ }^{14-17}$ a systematic characterization of serrated polyps in this population has not been hitherto reported. We hypothesized that serrated polyps in patients with inflammatory bowel disease may have similar biologic characteristics to sporadic serrated polyps and therefore undertook a retrospective review of colorectal serrated polyps diagnosed over a 14-year period among inflammatory bowel disease patients undergoing routine colonoscopic surveillance at our institution. Our aim was to characterize their histologic, clinicopathologic, and molecular features, and determine the associated risk of neoplasia development.

\section{Materials and methods}

\section{Cases and Patients}

Colorectal serrated polyps (index serrated polyps) diagnosed histologically between 2000 and 2013 were identified by query of our comprehensive electronic Gastrointestinal Pathology Division database (maintained prospectively by $\mathrm{NH}$ ) and by a retrospective, text-based search of our departmental pathology database for the term serrated combined with the terms inflammatory bowel disease, Crohn's disease, or ulcerative colitis. Inclusion criteria included patients with medically documented colonic inflammatory bowel disease undergoing surveillance colonoscopy in our institution and a confirmed diagnosis of serrated polyp of the large intestine between 2000 and 2013. Exclusion criteria included unavailability of archived slides for review and serrated polyps with high-grade dysplasia (one case).

H\&E-stained sections of all serrated polyps were reviewed independently by two gastrointestinal pathologists (ADP and $\mathrm{NH}$ ) who were blinded to original diagnoses and patient outcomes. Serrated polyps were evaluated for the type of serration present (e.g., resembling hyperplastic polyp vs sessile serrated adenoma/polyp) according to WHO criteria and were graded as positive, negative, or indefinite for dysplasia according to established criteria for the diagnosis of dysplasia in inflammatory bowel disease. ${ }^{18,19}$ Substantial inter-observer agreement was achieved $(\kappa=0.63$, s.e. $=0.08)$ and discordant cases were re-reviewed jointly for consensus. A separate control cohort of 28 inflammatory bowel disease patients diagnosed with conventional hyperplastic polyps during routine surveillance colonoscopy was selected randomly from the same patient registry. Clinicians performing surveillance colonoscopy on inflammatory bowel disease patients at our institution generally follow published guidelines, obtaining four-quadrant biopsies every $10 \mathrm{~cm}$.

Clinical information regarding the patients' age, sex, type and duration of inflammatory bowel disease, number of colonoscopic examinations, and the size, location, and endoscopic appearance of the serrated polyps was obtained from electronic medical records, and endoscopy and pathology reports. Polyp size was determined based on histologic measurements in all cases. Right-sided lesions were defined as those located proximal to the splenic flexure. The degree or extent of endoscopic or histologic active inflammation was not included in the analysis.

For each patient, endoscopy and pathology reports from all preceding and subsequent colonoscopic examinations and surgical resections were reviewed and all prior, synchronous, and metachronous diagnoses of dysplasia or carcinoma (prevalent neoplasia) were recorded. Sporadic adenomas, i.e., those occurring outside the documented area of colitis, as well as adenoma-like dysplastic polyps occurring within the area affected by colitis (classified as such based on established endoscopic and histologic criteria ${ }^{20}$ ), were not included as prevalent neoplasia, but the 11 patients with such sporadic adenomas were included in the analysis of incident advanced neoplasia. For the determination of risk of incident advanced neoplasia (high-grade dysplasia and carcinoma), developing after the diagnosis of each index serrated polyp (Supplementary Table 1), 12 patients in whom dysplasia or carcinoma had occurred before or at the same time as the index serrated polyp diagnosis and 17 patients with no follow-up colonoscopy were excluded. Rates were compared with a cohort of 1465 inflammatory bowel disease patients (from the same source population) with no baseline dysplasia, undergoing colonoscopic surveillance at our institution as previously described, ${ }^{21,22}$ after excluding patients with serrated or hyperplastic polyps.

This study was approved by the Institutional Review Board of the Icahn School of Medicine at Mount Sinai.

\section{Molecular Analysis}

Genomic DNA was extracted from formalin-fixed, paraffin-embedded tissue (10 unstained sections, each $5 \mu \mathrm{m}$ thick). DNA from each index lesion was extracted using the Maxwell Clinical Sample Concentrator instrument and Maxwell FFPE Plus LEV DNA purification kit (Promega, Madison, WI, 
USA) according to the manufacturer's recommended protocol. Multiplex PCR was carried out to amplify interested sequences of KRAS (exon 2), BRAF (exon 15), and PIK3CA (exons 9 and 20) genes. The primer sequences used and the expected size of the resulting PCR products were as follows:

KRAS E2 Forward (TTATAAGGCCTGCTGAAAA TGACTGAA) and KRAS E2 Reverse (TACCTCTATT GTTGGATCATATTCGT) yielding a 132 bp product; PIK3CA E9 Forward (GCTAGAGACAATGAATTAA GGG) and PIK3CA E9 Reverse (GAGATCAGCCAA ATTCAGTT) yielding a $186 \mathrm{bp}$ product; PIK3CA E20 Forward (TGGAATGCCAGAACTACAAT) and PIK3CA E20 Reverse (TTTGCCTGCTGAGAGTTA TT) yielding a $255 \mathrm{bp}$ product; and BRAF E15 Forward (CTCTTCATAATGCTTGCTCTGATAGG) and BRAF E15 Reverse (ATAGCCTCAATTCTTACC ATCCAC) yielding a $203 \mathrm{bp}$ product.

Amplified DNAs were mixed with 14 short specific DNA sequences (TAG primers) for each of the wild type or mutated sequences (Supplementary Table 2). When the target sequence is present, the primer binds and extends through allele-specific primer extension (ASPE). During ASPE, a reporter label (biotin-labeled dCTP) is incorporated. Extended TAG primers are then detected by specific anti-TAG sequences, each conjugated to differentially color-coded beads. Owing to the proximity of the DNA sequences involved, the PIK3CA-E9-Codon 542 WT and PIK3CA-E9-Codon 545 WT TAG primers used were identical. The reporter is streptavidin conjugated with R-phycoerythrin which is detected using the Luminex xMAP system (Luminex, Austin, TX, USA), according to the manufacturer's protocol. Mutations detected included KRAS codon 12 (GGT > AGT, TGT, CGT, GAT, GTT, or GCT), KRAS E2 codon 13 (GGC> TGC, AGC, CGC, GAC, GTC, or GCC), PIK3CA codon 542 (GAA >AAA), PIK3CA codon 545 (GAG $>$ AAG), PIK3CA codon 1047 (CAT $>$ CGT), and BRAF codon 600 (GTG > GAG, or AAG).

\section{Statistical Analysis}

Clinicopathologic characteristics were coded as either categorical (inflammatory bowel disease type, anatomic location) or dichotomous (sex, presence of dysplasia/carcinoma) variables, and frequencies were examined by type of serrated polyp (positive for low-grade dysplasia, indefinite for dysplasia, negative for dysplasia, hyperplastic polyp) with a chi square using the Fisher exact test for statistical significance. Continuous measures (age at diagnosis, size of polyp, number of colonoscopies, and length of colonoscopic surveillance period in months) were evaluated in relation to polyp type using an unbalanced two-way ANOVA design. We generated Kaplan-Meier curves to test for differences in the time to development of advanced neoplasia (highgrade dysplasia or adenocarcinoma) among patients with different types of index serrated polyps. Patients in whom neoplasia occurred prior to the diagnosis of the index serrated polyp were excluded from this part of the analysis. The Wilcoxon test was used to test for equality across strata. $P$-values less than 0.05 were considered statistically significant. All analyses were performed using SAS version 9.2 (SAS Institute, Cary, NC, USA).

\section{Results}

Serrated polyps were identified in 78 of 6602 inflammatory bowel disease patients (3453 women and 3149 men) undergoing surveillance colonoscopy in our institution during a 14-year period (2000-2013) for an overall prevalence rate of $1.2 \%$ (Table 1). Study patients had undergone a mean 8.2 colonoscopic examinations (range: 1-26) over a mean surveillance period of 101.5 months (range: 0-228 months). At least 65 (83\%) of the identified serrated polyps had been described by the endoscopist as a polyp, nodule, lesion, or fold (5 of these polyps were identified using chromoendoscopy), and all but $2(97 \%)$ occurred in areas with clinicopathologically documented involvement by inflammatory bowel disease.

Thirty-five $(45 \%)$ serrated polyps were negative for dysplasia and contained elongated crypts with hyperserrated mucinous epithelium along their entire length and basal dilatation or horizontal growth, features characteristic of sporadic sessile serrated adenoma/polyps ${ }^{19}$ (Figures 1a and b). Twenty-five $(32 \%)$ serrated polyps were positive for low-grade dysplasia and all contained complex, hyperserrated dysplastic epithelium with ectopic crypt formation, pencillate hyperchromatic nuclei and hypereosinophilic cytoplasm, fulfilling WHO criteria for sporadic traditional serrated adenomas ${ }^{19}$ (Figures 1c and d). Occasional serrated polyps positive for low-grade dysplasia contained admixed non-dysplastic serrated elements, as commonly occurs in sporadic traditional serrated adenomas. Eighteen $(23 \%)$ serrated polyps, classified as indefinite for dysplasia, comprised a mixed group with varied architectural features, some more closely resembling sessile serrated adenoma/polyps, others more closely resembling hyperplastic polyps, and others resisting classification, the common feature being serrated epithelium combined with foci of epithelial atypia deemed to fall short of a diagnosis of dysplasia. (Figures 1e and f). Examples of the endoscopic appearances of serrated polyps in the study are shown in Figure 2.

There were no statistically significant differences among patients with serrated polyps with respect to mean age, inflammatory bowel disease type, percentage with disease duration greater than 10 years, mean surveillance period, or mean number of colonoscopies per patient (Table 1). However, serrated polyps negative for dysplasia were more 
Table 1 Clinicopathologic features of study patients with serrated polyps

\begin{tabular}{|c|c|c|c|c|c|}
\hline & $S P-L G D(\mathrm{~N}=25)$ & $S P-I N D(\mathrm{~N}=18)$ & $S P-N E G(\mathrm{~N}=35)$ & $H P(\mathrm{~N}=28)$ & Reference $(\mathrm{N}=1465)$ \\
\hline Mean age \pm s.d. (years) & $56.4 \pm 14.4$ & $55.9 \pm 13.0$ & $54.3 \pm 14.2$ & $55.6 \pm 13.7$ & $37.5 \pm 16.9$ \\
\hline \multicolumn{6}{|l|}{ Sex } \\
\hline $\mathrm{M}(\%)$ & $16(64 \%)$ & $15(83 \%)$ & $16(46 \%)$ & $17(61 \%)$ & $737(50 \%)$ \\
\hline $\mathrm{F}(\%)$ & $9(36 \%)$ & $3(17 \%)$ & $19(54 \%)^{*}$ & $11(39 \%)$ & $728(50 \%)$ \\
\hline \multicolumn{6}{|l|}{ Polyp site } \\
\hline $\mathrm{R}(\%)$ & $5(20 \%)$ & $4(22 \%)$ & $20(57 \%)^{\#}$ & $9(32 \%)$ & \\
\hline $\mathrm{L}(\%)$ & $20(80 \%)$ & $14(78 \%)$ & $15(43 \%)$ & $19(68 \%)$ & \\
\hline Mean size \pm s.d. $(\mathrm{cm})$ & $1.23 \pm 0.80$ & $0.66 \pm 0.55$ & $0.87 \pm 0.45$ & $0.52 \pm 0.25^{+}$ & \\
\hline IBD type (UC:CD:IC) & 18:6:1 & $16: 2: 0$ & $22: 11: 2$ & 19:9:0 & 1416:49:0 \\
\hline Disease > 10 years $(\%)$ & $84 \%$ & $89 \%$ & $86 \%$ & $89 \%$ & $60 \%$ \\
\hline Mean surveillance (mo) & 101.1 & 98.7 & 103.2 & 113.4 & 88.0 \\
\hline Mean colonoscopies & 9.6 & 8.8 & 7.0 & 7.4 & 5.8 \\
\hline
\end{tabular}

Abbreviations: CD, Crohn's disease; F, female; HP, hyperplastic polyps; IBD, inflammatory bowel disease; IC, indeterminate colitis; L, left colon; M, male; mo, months; R, right colon (proximal to splenic flexure); s.d., standard deviation; SP-IND, serrated polyps indefinite for dysplasia; SP-LGD, serrated polyps positive for low-grade dysplasia; SP-NEG, serrated polyps negative for dysplasia; UC, ulcerative colitis. $P$-values were calculated for the indicated polyp type vs all other polyps in this study combined; all other $P$-values (not shown) were not significant.

${ }^{*} P=0.03$ vs other SPs and HPs in this study; ${ }^{\#} P=0.001$ vs other SPs and HPs in this study; ${ }^{+} P=0.009$ vs other SPs in this study.

common in women $(P=0.03)$ and were mostly right sided $(P=0.001)$, whereas serrated polyps positive for low-grade dysplasia and serrated polyps indefinite for dysplasia predominated in males and were more commonly left sided. The control cohort of inflammatory bowel disease patients with conventional hyperplastic polyps was statistically similar to the entire cohort with serrated polyps with respect to mean age, sex distribution, anatomic location, inflammatory bowel disease type, disease duration, mean surveillance period, and number of examinations. Hyperplastic polyps were smaller on average than other serrated polyps $(P=0.009)$.

Sequencing analysis for mutations in the KRAS (codons 12 and 13), BRAF (V600E/K), and PIK3CA (E542K, E545K, and H1047R) genes was undertaken for 42 serrated polyps where material was available and genomic DNA yield was adequate (Table 2). The majority of serrated polyps negative for dysplasia had mutations in BRAF $(P<0.001)$ whereas few serrated polyps positive for low-grade dysplasia and no serrated polyps indefinite for dysplasia had $B R A F$ mutations. In contrast, KRAS mutations were the most common mutations in serrated polyps positive for low-grade dysplasia and serrated polyps indefinite for dysplasia, but were only seen in a minority of serrated polyps negative for dysplasia. No mutations were identified in PIK3CA or BRAF V600K. KRAS and $B R A F$ mutations were mutually exclusive in all cases.

The association of serrated polyps with conventional (non-serrated) dysplasia and carcinoma was evaluated in two ways. First, the rate of prior, synchronous or metachronous dysplasia (any grade) or carcinoma (prevalent neoplasia) was determined over the entire duration of surveillance per patient (Table 3). Sporadic adenomas and adenoma-like dysplastic polyps (as defined in Materials and Methods) occurred in 11 of 106 patients in our study $(10.4 \%)$ and were not included in this analysis of prevalent neoplasia. Four of $35(11 \%)$ inflammatory bowel disease patients with serrated polyps negative for dysplasia were diagnosed with non-serrated dysplasia or carcinoma during a mean surveillance period of 103.2 months and a mean 7.0 colonoscopies per patient. In contrast, 19 of 25 (76\%) patients with serrated polyps positive for low-grade dysplasia and 7 of $18(39 \%)$ patients with serrated polyps indefinite for dysplasia were diagnosed with dysplasia or carcinoma during a similar mean surveillance interval and a similar mean number of colonoscopies $(P<0.001)$. In the majority of these patients $(74 \%)$, neoplastic lesions were identified in the same colonic segment as the index serrated polyp and in 7 of $19(37 \%)$ patients with serrated polyps positive for low-grade dysplasia, prevalent neoplasia consisted of a persistent serrated lesion with lowgrade dysplasia (i.e., an incompletely resected or recurrent lesion). By comparison, 6 of 28 (21\%) patients with conventional hyperplastic polyps had dysplasia or carcinoma, but this was not statistically significantly different from patients with serrated polyps negative for dysplasia and likely reflects the underlying neoplasia risk in this patient population.

Second, we examined the risk of incident advanced neoplasia (high-grade dysplasia and adenocarcinoma) developing among 77 patients with index serrated polyps (including hyperplastic polyps) and no prior or synchronous dysplasia or carcinoma (12 patients in whom any grade dysplasia or carcinoma had occurred before or at the same time as the index serrated polyp diagnosis and 17 patients with no follow-up colonoscopy were excluded). These were compared with a reference cohort of 

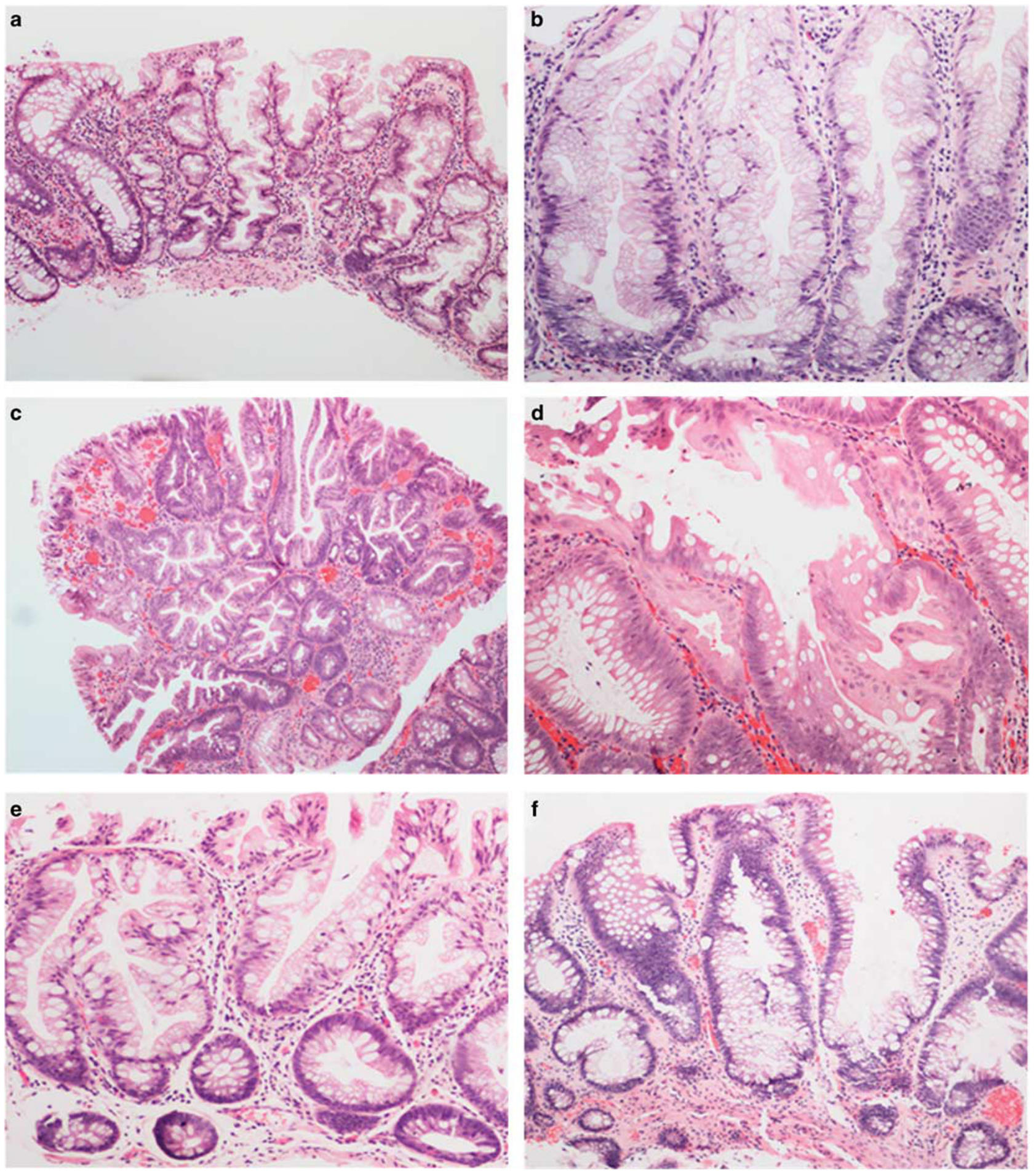

Figure 1 Photomicrographs of H\&E-stained sections from serrated polyps histologically classified as (a and b) negative for dysplasia; (c and $\mathbf{d}$ ) positive for low-grade dysplasia; and (e and f) indefinite for dysplasia (original magnification: $\times 200$ ). H\&E, hematoxylin and eosin.

1465 inflammatory bowel disease patients with no dysplasia at baseline, undergoing colonoscopic surveillance (Table 1 and Figure 3). Among 27 patients with index serrated polyps negative for dysplasia and no prior diagnosis of dysplasia, only $1(4 \%)$ developed conventional low-grade dysplasia and none developed advanced neoplasia during the follow-up period. In contrast, among 21 patients with index serrated polyps positive for low-grade dysplasia, 3 (14\%) developed metachronous highgrade dysplasia and 1 (5\%) developed adenocarcinoma. Of 11 patients with index serrated polyps 

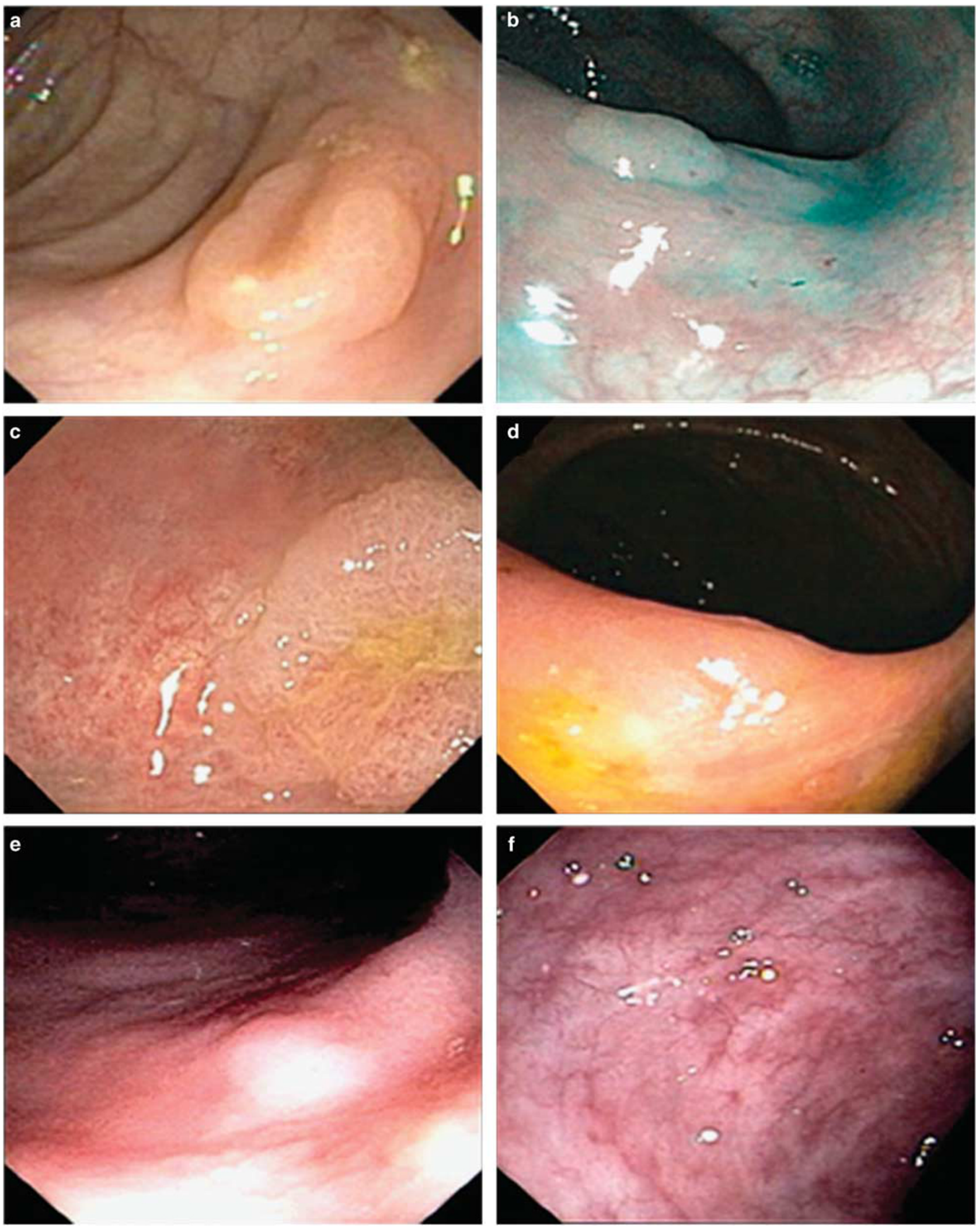

Figure 2 Endoscopic images of serrated colorectal polyps classified as negative for dysplasia: a 2-cm sessile polyp in the transverse colon (a) and a 5-mm flat polyp at $70 \mathrm{~cm}$ (b); positive for low-grade dysplasia: a 1-2-cm irregular sessile polyp with central umbilication at $40 \mathrm{~cm}$ (c) and a 6-7-mm slightly raised lesion in the ascending colon (d); and indefinite for dysplasia: a diminutive nodule in the ascending colon (e) and an ill-defined irregular raised area in the sigmoid colon (f) (courtesy of: James George, MD, Steven H Itzkowitz, MD, Mejdi Ahmad, MD, and Neville D Bamji, MD). 
Table 2 Molecular characteristics of serrated polyps

\begin{tabular}{|c|c|c|c|c|}
\hline & $S P-L G D(\mathrm{~N}=11)$ & $S P-I N D(\mathrm{~N}=8)$ & $S P-N E G(\mathrm{~N}=23)$ & Total $(\mathrm{N}=42)$ \\
\hline$B R A F$ mutations (V600E) (\%) & $2(18 \%)$ & $0(0 \%)$ & $15(65 \%)^{*}$ & $17(40 \%)$ \\
\hline KRAS mutations (exon E2) (\%) & $5(45 \%)$ & $2(25 \%)$ & $4(17 \%)$ & $11(26 \%)$ \\
\hline Wild type for genes tested (\%) & $4(36 \%)$ & $6(75 \%)$ & $4(17 \%)$ & $14(33 \%)$ \\
\hline
\end{tabular}

Abbreviations: SP-IND, serrated polyps indefinite for dysplasia; SP-LGD, serrated polyps positive for low-grade dysplasia; SP-NEG, serrated polyps negative for dysplasia. $P$-values were calculated for the indicated polyp type vs all other polyps in this study combined; all other $P$-values (not shown) were not significant.

${ }^{*} P<0.001$ vs non-BRAF mutated.

Table 3 Highest grade prevalent neoplasia per patient and serrated polyp type

\begin{tabular}{|c|c|c|c|c|c|}
\hline & $S P-L G D(\mathrm{~N}=25)$ & $S P-I N D(\mathrm{~N}=18)$ & $S P-N E G(\mathrm{~N}=35)$ & $H P(\mathrm{~N}=28)$ & Total $(\mathrm{N}=106)$ \\
\hline No neoplastic events & $6(24 \%)$ & $11(61 \%)$ & $31(89 \%)$ & $22(79 \%)$ & $70(66 \%)$ \\
\hline Low-grade dysplasia & $13(52 \%)$ & $4(22 \%)$ & $3(9 \%)$ & $2(7 \%)$ & $22(21 \%)$ \\
\hline High-grade dysplasia & $4(16 \%)$ & $2(11 \%)$ & $0(0 \%)$ & $2(7 \%)$ & $8(8 \%)$ \\
\hline Adenocarcinoma & $2(8 \%)$ & $1(6 \%)$ & $1(3 \%)$ & $2(7 \%)$ & $6(6 \%)$ \\
\hline
\end{tabular}

Abbreviations: HP, hyperplastic polyps; SP-IND, serrated polyps indefinite for dysplasia; SP-LGD, serrated polyps positive for low-grade dysplasia; SP-NEG, serrated polyps negative for dysplasia.

$P<0.001$.

indefinite for dysplasia, 2 (18\%) developed conventional low-grade dysplasia and $1(9 \%)$ had a highgrade dysplasia lesion during follow-up. Finally, among 18 patients with hyperplastic polyps, 2 (11\%) developed a lesion with conventional low-grade dysplasia and $1(6 \%)$ developed high-grade dysplasia. The 11 patients with sporadic adenomas or adenoma-like dysplastic lesions did not develop advanced neoplasia. As shown in Figure 3 (top panel), there were no statistically significant differences in actuarial 10-year rates of advanced neoplasia development between patients with index serrated polyps positive for low-grade dysplasia $(17 \%)$, serrated polyps indefinite for dysplasia $(8 \%)$, and the reference cohort with low-grade dysplasia at baseline (23\%). Similarly, Figure 3 (bottom panel) shows that the actuarial 10-year rate of advanced neoplasia for patients with index serrated polyps negative for dysplasia $(0 \%)$ was not significantly different from that of the reference cohort without dysplasia at baseline $(5 \%)$ or the group with index hyperplastic polyps (6\%). Overall, patients with index serrated polyps positive for lowgrade dysplasia had significantly shorter advanced neoplasia-free survival times compared with patients with index serrated polyps negative for dysplasia and index hyperplastic polyps $(P=0.02)$, whereas those with index serrated polyps indefinite for dysplasia fell in-between these groups.

\section{Discussion}

The morphologic, clinical, and molecular characteristics of sporadic colorectal serrated polyps are well-recognized, ${ }^{2-5,8,23}$ and have resulted in consensus recommendations for short- and long-term patient management. ${ }^{7,24,25}$ The present study addresses the dearth of available data regarding such lesions in patients with inflammatory bowel disease by means of a retrospective evaluation of serrated polyps diagnosed during the course of endoscopic surveillance. A low prevalence of serrated polyps in the setting of inflammatory bowel disease, $1.2 \%$ in our cohort, might account for the relative lack of attention devoted hitherto to serrated polyps in the inflammatory bowel disease literature. A recent Mayo Clinic study has corroborated this, reporting a combined prevalence of $2.1 \%$ for serrated epithelial changes (flat and polypoid serrated lesions) in inflammatory bowel disease patients. ${ }^{14}$

Histologic assessment of the serrated polyps in our cohort resulted in three groups: serrated polyps negative for dysplasia which lacked dysplasia and resembled sporadic sessile serrated adenoma/ polyps; serrated polyps positive for low-grade dysplasia which featured low-grade dysplasia and resembled sporadic traditional serrated adenomas; and serrated polyps indefinite for dysplasia, a heterogeneous group with mild cytological atypia that failed to meet criteria for low-grade dysplasia. Of the demographic and clinicopathologic characteristics associated with these polyps, the only ones that correlated with the likelihood of prevalent neoplasia or a future diagnosis of advanced neoplasia were sex and colonic location. Serrated polyps negative for dysplasia, which exhibited $11 \%$ rate of prevalent neoplasia and $0 \%$ 10-year actuarial rate of incident advanced neoplasia (similar to that of the dysplasiafree at baseline control cohort), predominated in 

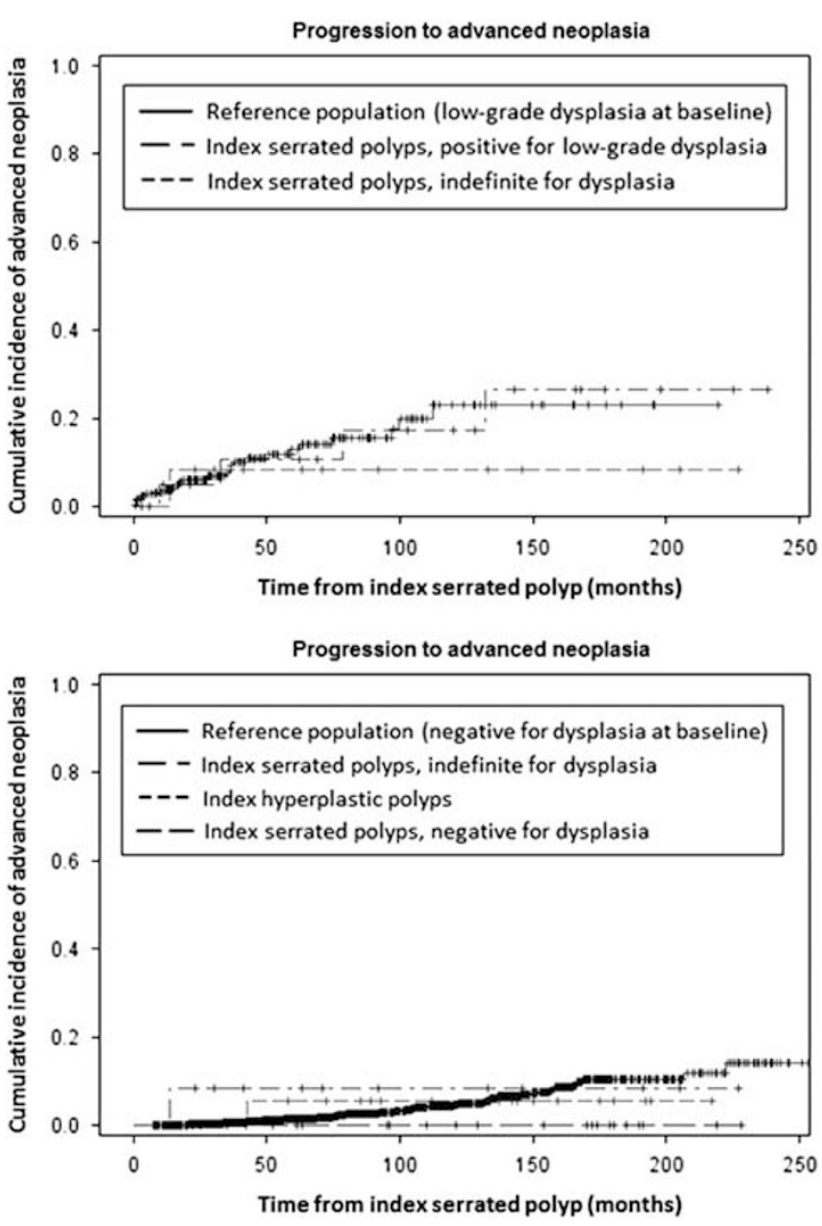

Figure 3 Kaplan-Meier curves showing cumulative incidence of advanced neoplasia (high-grade dysplasia or carcinoma) as a function of time (in months) from diagnosis of the indicated type of index serrated polyp compared with a reference cohort from the same overall population with inflammatory bowel disease undergoing surveillance colonoscopy in our institution. The reference cohort in the bottom panel comprised all patients $(N=1465)$ negative for dysplasia at baseline, whereas the reference cohort in the top panel comprised of patients $(N=216)$ with conventional (i.e., non-serrated), colitis-related low-grade dysplasia at baseline. Cross hatches (+) indicate censoring of patients with no further follow-up; vertical (step) lines indicate advanced neoplasia events.

females and in the right colon. By contrast, serrated polyps positive for low-grade dysplasia, with $76 \%$ rate of prevalent neoplasia and 17\% 10-year actuarial rate of incident advanced neoplasia (comparable to the $23 \%$ rate in our control cohort of inflammatory bowel disease patients with conventional, nonserrated, low-grade dysplasia at baseline), exhibited preponderance in males and in a left-sided colonic location. Other characteristics including mean age, inflammatory bowel disease type, disease duration, mean surveillance period, and mean number of colonoscopies were similar across polyp types, suggesting that our data were not significantly affected by selection or detection bias.

The rates of BRAF and KRAS mutations in serrated polyps negative for dysplasia and serrated polyps positive for low-grade dysplasia in our study closely resembles the rates reported in sporadic sessile serrated adenoma/polyps and traditional serrated adenomas, respectively., ${ }^{4,5}, 26$ This observation, in conjunction with their corresponding sex and anatomical site disparities, suggests that biologic parallels exist with sporadic sessile serrated adenoma/polyps and traditional serrated adenomas, despite the origin of at least $97 \%$ of our serrated polyps within chronically diseased inflammatory bowel disease mucosa. Although supervening cytologic dysplasia has been reported in up to $15 \%$ of sporadic sessile serrated adenoma/polyps, ${ }^{27,28}$ we did not encounter this phenomenon in our study, where serrated polyps with cytologic dysplasia histologically resembled sporadic traditional serrated adenomas. However, the possibility that this might be identified in a larger series cannot be discounted.

In order to capture all patients with serrated polyps, our study did not exclude 11 patients with sporadic adenomas and adenoma-like dysplastic polyps, but we did not include these adenomas in the calculations of prevalent dysplasia (Table 3) on the presumption that they were not relevant to inflammatory bowel disease-associated neoplasia. With respect to neoplastic progression, these 11 patients were not ignored, rather the adenomas themselves did not contain advanced neoplasia and none of the patients with these adenomas developed advanced neoplasia; hence, their inclusion did not influence our conclusions regarding the risk of advanced neoplasia development in inflammatory bowel disease patients with index serrated polyps. Even though the number of cases in our study is small, it seems that sporadic adenomas (occurring outside the area of colitis) and adenoma-like dysplastic polyps (occurring in an area of colitis, but resembling sporadic adenomas endoscopically and histologically) do not appear to impart any additional risk of neoplastic development on top of serrated polyps.

Little attention has been given hitherto to the pathology and possible clinical significance of serrated polyps indefinite for dysplasia in the setting of inflammatory bowel disease. Despite their heterogeneity, the lesions in this group demonstrated similarities to serrated polyps positive for lowgrade dysplasia with respect to predominance of male sex, left-sided colonic location, and KRAS mutations. These characteristics, combined with a $39 \%$ rate of prevalent neoplasia and a rate of incident advanced neoplasia development intermediate between serrated polyps negative for dysplasia and serrated polyps positive for low-grade dysplasia, support a potential role for serrated polyps indefinite for dysplasia in inflammatory bowel disease-related neoplasia which warrants further study.

Previous observations regarding serrated lesions in the colonic mucosa of patients with inflammatory bowel disease adjacent to or in association with adenocarcinoma have led to the conjecture that they might be neoplastic or preneoplastic in nature. Rubio 
et al. ${ }^{16}$ described higher rates of dysplastic serrated lesions near carcinomas in resection specimens from inflammatory bowel disease patients compared with non-inflammatory bowel disease patients. Kilgore et al. ${ }^{15}$ reported non-dysplastic serrated lesions in $33 \%$ of cancer-bearing resection specimens from patients with Crohn's disease compared with $10 \%$ of controls without cancer. Srivastava et al. ${ }^{17}$ described patients with longstanding inflammatory bowel disease and multiple hyperplastic polyps and sessile serrated adenoma/polyps (reminiscent of the serrated polyposis syndrome), nearly half of which contained KRAS but not BRAF mutations. They reported that cancers developed in two patients with conventional adenomatous dysplasia or traditional serrated adenomas but not in a third patient with non-dysplastic serrated lesions. More recently, a study of serrated epithelial changes in patients with inflammatory bowel disease, evidently comprising both raised and flat lesions, reported a cumulative incidence of subsequent colorectal neoplasia of $30 \%$ at 3 years compared with $9 \%$ in control patients, but the difference was not significant when history of prior or synchronous dysplasia was taken into account. ${ }^{14}$ Bossard et al. ${ }^{29}$ reported that serrated polyps, including hyperplastic polyps and sessile serrated adenoma/polyps, were present in $7 \%$ of patients with inflammatory bowel disease-related neoplasia and that $B R A F$ mutations occurred only in some of them, whereas KRAS mutations were restricted to non-serrated dysplastic lesions.

The present study is, to our knowledge, the first systematic and comprehensive clinicopathologic, histologic, and molecular characterization of serrated polyps in patients with inflammatory bowel disease and underscores a strong association with neoplasia development in a subset of these lesions. Dysplasia in these polyps was blindly and independently evaluated by two gastrointestinal pathologists with good inter-observer agreement and the rates of prevalent neoplasia and incident advanced neoplasia were compared with control groups and reference population cohorts. However, there are several limitations to our study. First, it is a retrospective analysis of previously identified serrated polyps with no assurance that data collected from patient medical records was entirely reliable or complete. Second, there is a possible referral bias since many patients come to our institution specifically for the management of dysplasia in the setting of inflammatory bowel disease. Third, determination of polyp size by histologic measurement is intrinsically inaccurate. Fourth, we could not determine whether completeness of endoscopic removal of these polyps was uniformly achieved or even attempted. Fifth, we were able to extract $B R A F$ and $K R A S$ mutation status information in only about half (54\%) of the serrated polyps in our study, but we have no indication to suspect that these were not overall representative samples. Finally, comparison of the risks of neoplasia development among patients with varying surveillance examination intervals is inherently inexact, although we determined that these parameters were statistically similar across all polyp types.

We conclude that the clinical, pathologic, and biologic characteristics of serrated polyps in patients with inflammatory bowel disease resemble those of their sporadic counterparts and are associated with the presence or absence of dysplasia. Additionally, whereas serrated polyps that lack dysplasia seem to pose little additional risk of associated neoplasia, those with low-grade dysplasia, and to a lesser degree those indefinite for dysplasia, are associated with increased rates of synchronous and metachronous neoplasia. Although it is reasonable to conclude that a diagnosis of low-grade dysplasia or indefinite for dysplasia in an inflammatory bowel disease-associated serrated polyp warrants clinical caution and careful follow-up, further studies will be needed to translate these conclusions into specific clinical recommendations.

\section{Disclosure/conflict of interest}

The authors declare no conflict of interest.

\section{References}

1 Snover DC. Update on the serrated pathway to colorectal carcinoma. Hum Pathol 2011;42:1-10.

2 Sweetser S, Smyrk TC, Sinicrope FA. Serrated colon polyps as precursors to colorectal cancer. Clin Gastroenterol Hepatol 2013;11:760-767.

3 East JE, Saunders BP, Jass JR. Sporadic and syndromic hyperplastic polyps and serrated adenomas of the colon: classification, molecular genetics, natural history, and clinical management. Gastroenterol Clin North Am 2008;37:25-46.

4 Noffsinger AE. Serrated polyps and colorectal cancer: new pathway to malignancy. Annu Rev Pathol 2009;4: 343-364.

5 O'Brien MJ. Hyperplastic and serrated polyps of the colorectum. Gastroenterol Clin North Am 2007;36: 947-968.

6 Leonard DF, Dozois EJ, Smyrk TC et al. Endoscopic and surgical management of serrated colonic polyps. Br J Surg 2011;98:1685-1694.

7 Lieberman DA, Rex DK, Winawer SJ et al. Guidelines for colonoscopy surveillance after screening and polypectomy: a consensus update by the US Multi-Society Task Force on Colorectal Cancer. Gastroenterology 2012;143:844-857.

8 Rex DK, Ahnen DJ, Baron JA et al. Serrated lesions of the colorectum: review and recommendations from an expert panel. Am J Gastroenterol 2012;107: 1315-1329.

9 Farraye FA, Odze RD, Eaden J et al. AGA technical review on the diagnosis and management of colorectal neoplasia in inflammatory bowel disease. Gastroenterology. 2010;138:746-774.

10 Friedman S, Rubin PH, Bodian C et al. Screening and surveillance colonoscopy in chronic Crohn's colitis: 
results of a surveillance program spanning 25 years. Clin Gastroenterol Hepatol 2008;6:993-998.

11 Itzkowitz SH, Harpaz N. Diagnosis and management of dysplasia in patients with inflammatory bowel diseases. Gastroenterology 2004;126:1634-1648.

12 Harpaz N, Polydorides AD. Colorectal dysplasia in chronic inflammatory bowel disease: pathology, clinical implications, and pathogenesis. Arch Pathol Lab Med 2010;134:876-895.

13 Harpaz N, Ward SC, Mescoli C et al. Precancerous lesions in inflammatory bowel disease. Best Pract Res Clin Gastroenterol 2013;27:257-267.

14 Johnson DH, Khanna S, Smyrk TC et al. Detection rate and outcome of colonic serrated epithelial changes in patients with ulcerative colitis or Crohn's colitis. Aliment Pharmacol Ther 2014;39:1408-1417.

15 Kilgore SP, Sigel JE, Goldblum JR. Hyperplastic-like mucosal change in Crohn's disease: an unusual form of dysplasia? Mod Pathol 2000;13:797-801.

16 Rubio CA, Befrits R, Jaramillo E et al. Villous and serrated adenomatous growth bordering carcinomas in inflammatory bowel disease. Anticancer Res 2000;20: 4761-4764.

17 Srivastava A, Redston M, Farraye FA et al. Hyperplastic/serrated polyposis in inflammatory bowel disease: a case series of a previously undescribed entity. Am J Surg Pathol 2008;32:296-303.

18 Riddell RH, Goldman H, Ransohoff DF et al. Dysplasia in inflammatory bowel disease: standardized classification with provisional clinical applications. Hum Pathol 1983;14:931-968.

19 Snover DC, Ahnen DJ, Burt RW, Odze RD. Serrated polyps of the colon and rectum and serrated polyposis. In: Bosman FT CF, Hruban RH, Theise ND (eds). WHO Classification of Tumours of the Digestive System, 4th edn. International Agency for Research for Cancer: Lyon, France, 2010, pp 160-165.
20 Rubin PH, Friedman S, Harpaz N et al. Colonoscopic polypectomy in chronic colitis: conservative management after endoscopic resection of dysplastic polyps. Gastroenterology 1999;117:1295-1300.

21 Gupta RB, Harpaz N, Itzkowitz S et al. Histologic inflammation is a risk factor for progression to colorectal neoplasia in ulcerative colitis: a cohort study. Gastroenterology 2007;133:1099-1105.

22 Ullman T, Croog V, Harpaz N et al. Progression of flat low-grade dysplasia to advanced neoplasia in patients with ulcerative colitis. Gastroenterology 2003;125: 1311-1319.

23 Torlakovic E, Skovlund E, Snover DC et al. Morphologic reappraisal of serrated colorectal polyps. Am J Surg Pathol 2003;27:65-81.

24 Hiraoka S, Kato J, Fujiki S et al. The presence of large serrated polyps increases risk for colorectal cancer. Gastroenterology 2010;139:1503-1510.

25 Schreiner MA, Weiss DG, Lieberman DA. Proximal and large hyperplastic and nondysplastic serrated polyps detected by colonoscopy are associated with neoplasia. Gastroenterology 2010;139:1497-1502.

26 Aust DE, Baretton GB. Serrated polyps of the colon and rectum (hyperplastic polyps, sessile serrated adenomas, traditional serrated adenomas, and mixed polyps)proposal for diagnostic criteria. Virchows Arch 2010; 457:291-297.

27 Bettington M, Walker N, Clouston et al. The serrated pathway to colorectal carcinoma: current concepts and challenges. Histopathology 2013;62:367-386.

28 Lash RH, Genta RM, Schuler CM. Sessile serrated adenomas: prevalence of dysplasia and carcinoma in 2139 patients. J Clin Pathol 2010;63:681-686.

29 Bossard C, Denis MG, Bezieau S et al. Involvement of the serrated neoplasia pathway in inflammatory bowel disease-related colorectal oncogenesis. Oncol Rep 2007;18:1093-1097.

Supplementary Information accompanies the paper on Modern Pathology website (http://www.nature.com/ modpathol) 